\title{
Using 3D X-ray Microscopy to Study Crown Root Development and Primary Root Tip Growth in Diverse Maize (Zea mays L.) Lines
}

\author{
Keith E. Duncan ${ }^{1 *}$, Adam L. Bray ${ }^{1,2}$, Tyler G. Dowd ${ }^{1}$ and Christopher N. Topp ${ }^{1}$ \\ 1. Donald Danforth Plant Science Center, St. Louis, MO, USA. \\ 2. Division of Plant Sciences, University of Missouri, Columbia, MO, USA. \\ * Corresponding author: kduncan@danforthcenter.org
}

Plant anatomy and development are difficult to study if researchers are restricted to the examination of 2D images, regardless of the resolution of the imaging technology being used. Generating detailed 3D volumes of plant samples at cellular resolution would allow measurement and analysis of complicated plant cell biology in unprecedented detail. Electron microscopy (EM) allows high resolution imaging but there are practical limitations for 3D EM with regard to sample size and throughput [1]. There are excellent examples of researchers using conventional EM sample preparation techniques for improving contrast in X-ray microscopy (XRM) imaging of plant samples [2]. We have been adapting these protocols to take advantage of the relatively wide sample size range capabilities of the Zeiss Xradia 520 Versa to study plant cell biology with 3D XRM at near-EM level resolution.

Maize shoot-borne roots, also known as crown or brace roots, form near the outer layers of stem tissue and must break through stiff epidermal tissue in order to access water and nutrients in the soil. Additionally, the connections of crown root xylem and phloem are critical for the transport of water and carbon to rapidly growing tissue. We examined maize stalk samples at this vascular nexus to study crown root initiation and elongation as well as vascular development over a range of imaging resolutions with the XRM (Figs 1,2). We have also been documenting cell division and elongation in 3D for developing maize primary root tips, comparing early morphology between maize lines with demonstrated variation in elongation rates as well as overall root system architecture (Fig 3).

We are currently working on segmentation protocols with a variety of image analysis resources to reliably and accurately identify specific cells and tissues within these 3D volumes. This will allow measurement and statistical comparison of important root traits across diverse maize lines [3-5]. The computational aspect of our research is crucial to take advantage of the rich data sets provided by high resolution 3D imaging using XRM technology for the measurement and analysis of complicated biological structures $[6]$.

References:

[1] B Titze and C Genoud, Biol Cell 108 (2016), p. 307.

[2] YM Staedler, D Masson and J Schönenberger, PLoS One 8 (2013), p. e75295.

[3] YM Staedler et al., J Exper Bot 69 (2018), p. 525.

[4] J Jeiter et al., Int J Plant Sci 179 (2018), p.275.

[5] TPS Reynolds et al., J R Soc Interface 15 (2018), p. 20180144.

[6] This work is supported by collaborative agreements with Valent BioSciences and Sumitomo Chemical Company, and the National Science Foundation projects IIA-135540 and IOS-1638507. 

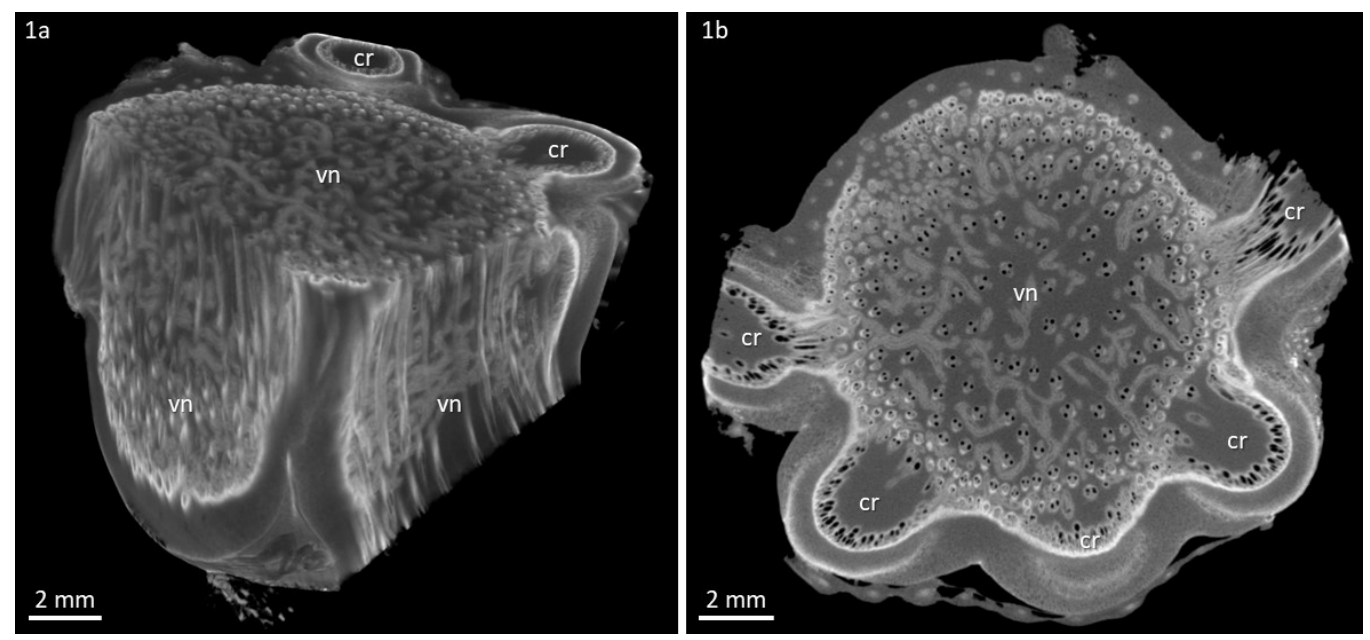

Figure 1. Maize stalk segment contrast enhanced with Lugol's iodine and X-ray microscope scanned with a $0.4 x$ objective lens. a. Screen shot of cropped 3D volume showing extensive vascular network (vn) associated with developing crown roots (cr). b. Single 2D image showing cross-sectional view through the vascular network in the region of crown root emergence.
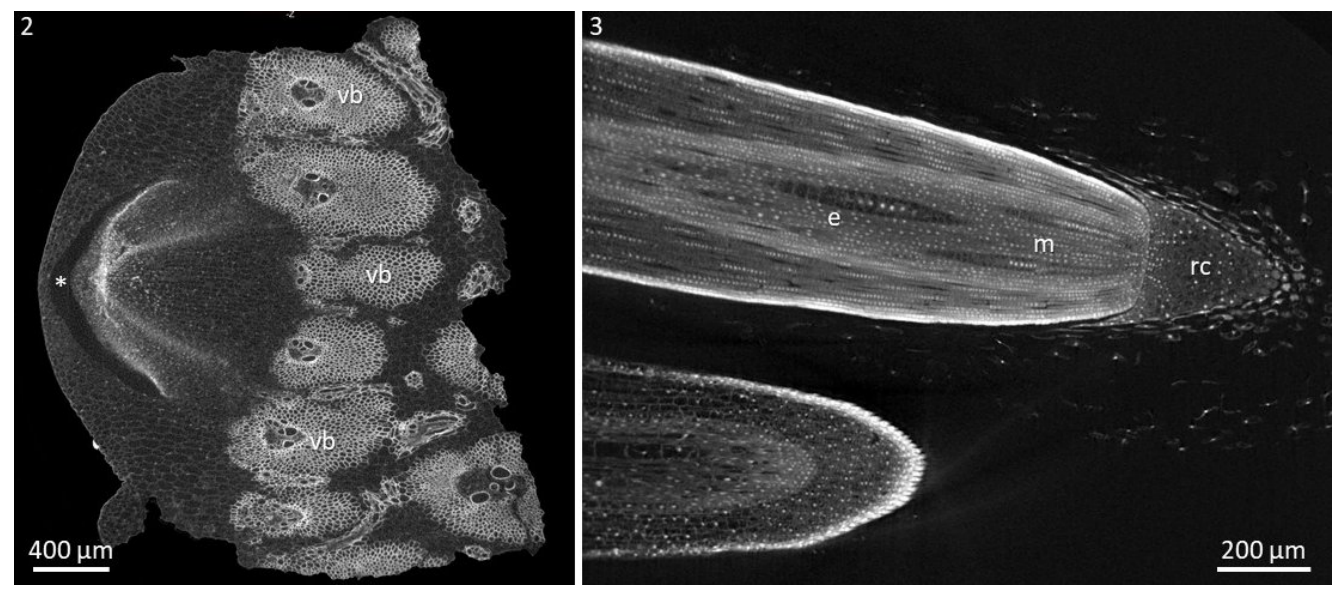

Figure 2. Digital slice through a maize crown root primordium with primordium tip (asterisk) and vascular bundles (vb); contrast enhanced with Lugol's iodine.

Figure 3. Digital slice through two maize root tips showing root cap (rc), and meristematic (m) and elongation zones (e); contrast enhanced with phosphotungstic acid in ethanol. 